\section{Abwehrrechte von Mitgesellschaftern einer Radiologiepraxis bei beabsichtigtem Verkauf an einen Investor}

\section{Einleitung}

In den vergangenen Jahren häufen sich Fälle, in denen Arztpraxen unterschiedlicher Fachrichtungen durch Kapitalinvestoren aufgekauft werden. Auch Radiologiepraxen sind hiervon betroffen. Der Gesetzgeber hat versucht, den Einfluss von Investoren auf Arztpraxen durch das GKV-Versorgungsstrukturgesetz (GKV-VStG) vom 22.12.2011 (BGBI. I, 2983) und das Terminservice- und Versorgungsgesetz (TSVG) vom 06.05.2019 (BGBI. 2019, 646) durch Erwerbsbeschränkungen einzudämmen. Diese Bemühungen hatten bisher allerdings nur einen mäßigen Erfolg, da über die Gründung von Medizinischen Versorgungszentren (MVZ) der Praxiserwerb durch Investoren auch weiterhin möglich ist. Ist eine Praxis als Berufsausübungsgemeinschaft (BAG) organisiert, kann dies zu Problemen führen, wenn die Gesellschafter unterschiedlicher Meinung darüber sind, ob die Praxis veräußert werden soll.

Dieser Beitrag soll aus gesellschafts- und vertragsarztrechtlicher Perspektive die Situation näher beleuchten, in der die Mehrheit der Mitglieder einer BAG beschließt, die Praxis insgesamt an ein MVZ zu veräußern, während eine Minderheit diesen Beschluss nicht mitträgt und sich durch diese, auch für sie bindende, Entscheidung benachteiligt sieht. Im Fokus sollen dabei insbesondere Abwehrmöglichkeiten der überstimmten Gesellschafter stehen, um die von der verkaufswilligen Mehrheit beabsichtigte Veräußerung zu verhindern oder aber die BAG aufrechtzuerhalten.

Es sei vorausgeschickt, dass für die rechtliche Beurteilung der dargestellten Situation insbesondere die konkrete Ausgestaltung des jeweiligen Gesellschaftsvertrages der BAG entscheidend ist. Diese beeinflusst maßgeblich die gesellschaftsrechtliche Lage. Im Rahmen der folgenden Betrachtung wird daher vorausgesetzt, dass es im Gesellschaftsvertrag der BAG eine Fortsetzungsklausel gibt, welche auch im Falle des Ausscheidens eines Gesellschafters eine
Fortsetzung der BAG durch neue Besetzung der vakant gewordenen Arztstelle in der BAG vorsieht.

\section{Gesellschaftsrechtliche Wirksamkeit des Mehrheits- beschlusses in einer BAG}

Die Beschlussfassung in einer Gesellschaft bürgerlichen Rechts (GbR), in der eine BAG häufig geführt wird ${ }^{1}$, ist in $\S 709$ Abs. 1 BGB geregelt. Danach stellt Einstimmigkeit das grundsätzliche Prinzip der Willensbildung dar. Ausnahmsweise hiervon können jedoch Mehrheitsentscheidungen getroffen werden, wenn dies im Gesellschaftsvertrag bezüglich bestimmter Gegenstände gestattet ist, §709 Abs. 2 BGB.

Wenn dagegen bei einer derartigen Beschlussfassung Gesellschafter überstimmt und damit in ihrem Willen übergangen werden, deren Interessen jedoch je nach Lage des Sachverhalts schutzwürdig sind, hat die zivilrechtliche Rechtsprechung Anforderungen entwickelt, die an die Wirksamkeit von Mehrheitsentscheidungen in Personengesellschaften zu stellen sind. Danach muss ein Mehrheitsbeschluss zumindest zwei Voraussetzungen erfüllen, um wirksam zu sein.

\section{Formelle Legitimation}

Nach der höchstrichterlichen Rechtsprechung bedarf der Mehrheitsbeschluss zunächst der sog. formellen Legitimation.

Das bedeutet, dass dem Text des Gesellschaftsvertrages zu entnehmen sein muss, dass über den betroffenen Gegenstand überhaupt mittels eines Mehrheitsbeschlusses - unter bewusster Abweichung vom grundsätzlichen Prinzip der Einstimmigkeit - entschieden werden darf bzw. soll.

1 Soweit eine BAG in der Rechtsform einer Partnerschaftsgesellschaft nach § 1 PartGG geführt wird, gelten die nachfolgenden Ausführungen weitgehend entsprechend. Die Besonderheiten der Partnerschaftsgesellschaft können allerdings im Rahmen dieses Beitrages nicht gesondert dargestellt werden.

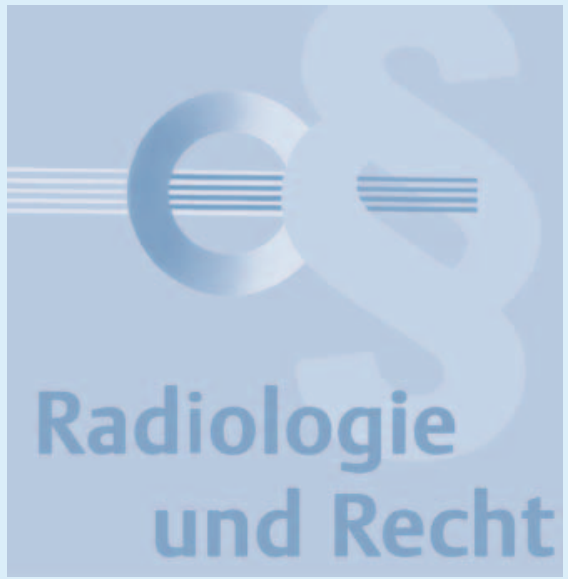

Eine minutiöse Auflistung dieser (lediglich) durch die Mehrheit der Gesellschafter wirksam zu beschließenden Gegenstände als Ausfluss eines etwaigen „Bestimmtheitsgrundsatzes" wird indes nicht gefordert. Es genügt vielmehr, wenn der Vertrag im Wege der nach allgemeinen Grundsätzen vorzunehmenden Auslegung (§§133, 157 $B G B)$ eindeutig ergibt, dass der betroffene Beschlussgegenstand der Mehrheitsentscheidung unterworfen sein soll. ${ }^{2}$ Die Auslegung des Vertrages geschieht dabei bei einer GbR bzw. BAG, welche eine Personengesellschaft darstellt, subjektiv. Das bedeutet, dass

„nicht allein auf den Wortlaut des (schriftlichen) Gesellschaftsvertrags abzustellen [ist], sondern [es] können auch außerhalb des Vertragstextes liegende Umstände für die Auslegung von Bedeutung sein [,] wie insbesondere die Entstehungsgeschichte der in Rede stehenden Bestimmungen des Gesellschaftsvertrags oder ein übereinstimmender Wille der Vertragsparteien “. ${ }^{3}$

Hinsichtlich der Beschlussgegenstände ist im Rahmen der Prüfung dieser formellen Legitimation keine Einschränkung zu machen. Zwar wurde früher noch angenommen, bereits die formelle Legitimation sei nicht

2 BGH, Urteil vom 21.10 2014, Az.: II ZR 84/ 13 = BGHZ 203, 77-98, juris Rn. 10, 14 ff., 24, jeweils m.w.N., unter Fortentwicklung der Rechtsprechung aus dem Urteil vom 15.01.2007, Az.: II ZR 245/05 = BGHZ 170, 283-299, „OTTO“, juris Rn. 9 m.w.N.; ebenso Urteil vom 24.11.2008, Az.: II ZR 116/ $08=$ BGHZ 179, 13-27, „Schutzgemeinschaftsvertrag II“, juris Rn. 15.

3 BGH, Urteil vom 21.10.2014, Az.: II ZR 84/13 (Fn. 2), juris Rn. 24 m.w.N. 
gegeben, wenn „außergewöhnliche“ Geschäfte oder Grundlagengeschäfte durch gesellschaftsvertragliche Regelung vom Einstimmigkeitsprinzip ausgenommen würden. ${ }^{4}$ Dies hatte zur Folge, dass derartige Mehrheitsbeschlüsse, welche solche Geschäfte betrafen, ohne weiteres von vornherein unwirksam waren.

Für eine derartige Einschränkung finden sich jedoch im Gesetz keine Anhaltspunkte, wie auch der Bundesgerichtshof nunmehr herausgestellt hat. Der Absatz 2 des $§ 709$ BGB sieht danach eine von der Privatautonomie der Vertragsparteien gedeckte Abweichungsmöglichkeit vom Einstimmigkeitsprinzip vor, gerade damit Vereinbarungen des Gesellschaftsvertrages im Sinne der Praktikabilität flexibel gestaltet werden können. ${ }^{5}$

Die Anforderung der formellen Legitimation beschreibt demnach - verkürzt ausgedrückt - lediglich das Erfordernis einer ausreichenden vertraglichen Grundlage für einen Mehrheitsbeschluss der Gesellschafter.

Entscheidet sich die Mehrheit der Gesellschafter einer gemeinschaftlich betriebenen Radiologiepraxis dafür, die Praxis zu veräußern, und trifft einen entsprechenden Mehrheitsbeschluss, so muss für diesen in formeller Hinsicht demnach zunächst eine ausreichende Grundlage nach o.g. Grundsätzen im Gesellschaftsvertrag vorhanden sein. Eine grundsätzliche Unzulässigkeit dieses Mehrheitsbeschlusses kommt nach den vorstehenden Grundsätzen keinesfalls in Betracht.

Somit ergibt sich die naheliegende Möglichkeit, einem sich aufgrund der Sachlage abzeichnenden Beschluss über die Veräußerung der Gemeinschaftspraxis, welcher durch lediglich die Mehrheit der Gesellschafter vorgenommen zu werden droht, im Vorfeld die formelle Legitimation eindeutig zu entziehen. Dies geschieht dadurch, dass in den Gesellschaftsvertrag von vornherein eine Klausel aufgenommen

4 So noch $B G H$, Urteil vom 12.10.1952, Az.: II ZR 260/51 = BGHZ 8, 35-47, juris Rn. 11, 13, jeweils m.w.N.

5 Vgl. BGH, „Schutzgemeinschaftsvertrag II“ (Fn. 2), juris Rn. 14, 16.; „OTTO“ (Fn. 2), juris Rn. 6. wird, welche bestimmt, dass ein Verkauf der Praxis ausschließlich durch einstimmigen Beschluss aller (vorhandenen) Gesellschafter der BAG möglich ist (dazu im Folgenden mehr).

\section{Materielle Legitimation}

In einem zweiten Schritt ist für die Wirksamkeit einer Mehrheitsentscheidung die sog. materielle Legitimation erforderlich. Diese stellt an den Inhalt des Beschlusses die Anforderung, dass keine Verletzung der gesellschaftsrechtlichen Treuepflicht der Mehrheit der Gesellschafter gegenüber der (überstimmten) Minderheit vorliegt. ${ }^{6}$

Auch auf dieser Stufe der gilt der Maßstab, der an einen solchen Beschluss anzulegen ist, für alle Beschlussgegenstände gleichermaßen, d. h. für gewöhnliche Geschäfte ebenso wie für außergewöhnliche ${ }^{7}$ sowie Grundlagengeschäfte oder Maßnahmen, die in unentziehbare Rechte der überstimmten Minderheit der Gesellschafter eingreifen ${ }^{8}$. Eine Beschränkung auf den sog. Kernbereich der Gesellschafterrechte wurde durch die jüngere höchstrichterliche Rechtsprechung schließlich aufgegeben. ${ }^{9}$

Greift ein Mehrheitsbeschluss in den „Kernbereich“ der Gesellschafterrechte (ergänzend hierzu im Folgenden) ein, hat dies lediglich noch zur Konsequenz, dass ein Treuepflichtverstoß der Mehrheitsgesellschafter vermutet wird, sodass diese darlegen und beweisen müssen, dass ein solcher Verstoß gerade nicht vorliegt. In allen anderen Fällen trifft die Darlegungs- und Beweis-

6 Vgl. BGH, Urteil vom 21.10.2014, Az.: II ZR 84/13 (Fn. 2), juris Rn. 11 ff.; „Schutzgemeinschaftsvertrag II“ (Fn. 2), juris Rn. 16f.; „OTTO” (Fn. 2), juris Rn. $9 \mathrm{f}$.

7 BGH, Urteil vom 21.10.2014, Az.: II ZR 84/13 (Fn. 2), juris Rn. 16; „Schutzgemeinschaftsvertrag II“ (Fn. 2), juris Rn. 17; für eine Beschränkung auf „gewöhnliche“ Geschäfte hingegen noch „OTTO“ (Fn. 2), juris Rn. 9 m.w.N. unter Berufung auf diverse Entscheidungen des Reichsgerichts.

8 BGH, „Schutzgemeinschaftsvertrag II“ (Fn. 2), juris Rn. 17.

9 BGH, Urteil vom 21.10.2014, Az.: II ZR 84/13 (Fn. 2), juris Rn. 13, 19 m.w.N.; "Schutzgemeinschaftsvertrag II“ (Fn. 2), juris Rn. 17; für eine Beschränkung auf den „Kernbreich“ im Grundsatz noch in „OTTO“ (Fn. 2), juris Rn. 9. last nach allgemeinen Grundsätzen die (überstimmte) Minderheit, welche eine derartige Treuepflichtverletzung behauptet. ${ }^{10}$

So ist nach der ständigen höchstrichterlichen Rechtsprechung für die materielle Legitimation einer Mehrheitsklausel im Gesellschaftsvertrag einer GbR

„letztlich maßgeblich immer [entscheidend], ob der Eingriff im Interesse der Gesellschaft geboten und dem betroffenen Gesellschafter unter Berücksichtigung seiner eigenen schutzwürdigen Belange zumutbar ist“. ${ }^{11}$

Den Eingriff stellt dabei der Mehrheitsbeschluss dar, welcher die überstimmte Minderheit in ihren Interessen übergeht und deren Rechte beschneidet. Angedeutet ist in jenem Urteil des Bundesgerichtshofs, dass die Entscheidung über die Zumutbarkeit im Wege einer Bilanzierung der aus dem Eingriff resultierenden Vor- und Nachteile erfolgen kann. ${ }^{12}$

Zusammengefasst folgt daraus, dass über die Prüfung einer formellen Berechtigung zur Willensbildung mittels eines Mehrheitsbeschlusses im Gesellschaftsvertrag hinaus der Beschluss selbst dahingehend zu überprüfen ist, ob dieser in die Rechte der überstimmten Gesellschafter in lediglich zumutbarer Weise eingreift und ob diese Beeinträchtigung durch überwiegende Interessen der Gesellschaft gerechtfertigt ist. Diese Grundsätze gelten für eine als GbR organisierte BAG ebenso wie für eine Praxis, welche als Partnerschaftsgesellschaft (PartG) geführt wird. Denn die Absätze 1 und 2 des $§ 709$ BGB sind über die Vorschrift des $\S 1$ Abs. 4 PartGG auch auf eine solche anwendbar.

Vor diesem Hintergrund ist demnach zu prüfen, ob ein in Frage stehender Mehrheitsbeschluss über den Verkauf einer

10 BGH, „Schutzgemeinschaftsvertrag I"“ (Fn. 2), juris Rn. 17; „OTTO“ (Fn. 2), juris Rn. 10 m.w.N.

11 BGH, Urteil vom 21.10.2014, Az.: II ZR 84/13 (Fn. 2), juris Rn. 19 m.w.N.; ebenso Urteil vom 04.07.2005, Az.: II ZR 354/03 = MDR 2005, 1421-1422, juris Rn. 31; Urteil vom 19.10.2009, Az.: II ZR 240/08 = BGHZ 183, 113, „Sanieren oder Ausscheiden“, juris Rn. 23.

12 Vgl. BGH, „Sanieren oder Ausscheiden“ (Fn. 11), juris Rn. 23. 
Gemeinschaftspraxis auch materiell legitimiert werden kann.

Es ist ersichtlich, dass ein Verkauf der Praxis als Sachgesamtheit dazu führt, dass nicht nur die verkaufswillige Mehrheit, sondern auch die überstimmte Minderheit sämtliche Sachmittel verliert, welche sie zur Ausübung ihrer ärztlichen Tätigkeit benötigt. Dabei ist insbesondere zu beachten, dass die Entscheidung der Gesellschafter zum Zusammenschluss in einer BAG eine spezifische Entscheidung hinsichtlich der ärztlichen Berufsausübung darstellt, welche durch Art. 12 Abs. 1 GG als solche besonders geschützt ist. Der Bundesgerichtshof führt hierzu aus:

„Diesem Schutz ist immanent, daß [sic] die Gemeinschaftspraxis in der Form und mit der Anzahl von Vertragsärzten grundsätzlich weiterbetrieben werden kann, die für sie vorgesehen ist."13

Dies wird zumindest im Bereich der vertragsärztlichen Berufsausübung unter den Bedingungen von Zulassungsbeschränkungen unter anderem daraus hergeleitet, dass eine Verkleinerung einer BAG durch das Ausscheiden eines Gesellschafters in $\S 103$ Abs. 6 SGB V erschwerten Bedingungen unterworfen werde. ${ }^{14}$ So bestimmt nämlich der Satz 2 der Vorschrift, dass die Interessen der in der Praxis verbleibenden Vertragsärzte im Rahmen des Ausschreibungs- und Nachbesetzungsverfahrens angemessen zu berücksichtigen sind.

Problematisch erscheint bei der vorliegend betrachteten Verkaufssituation, dass der Verkauf der Praxis ggf. auch die gleichzeitige, zulässige Beendigung der GbR bzw. der BAG bedeuten könnte. Daraus würde ersichtlich folgen, dass auch die BAG in ihrem Bestand nicht mehr schutzwürdig sein könnte.

Denn jeder Gesellschafter hat grundsätzlich das jederzeitige Recht zur Kündigung der Gesellschaft (§723 Abs. 1 S. 1 BGB). Beabsichtigt die Mehrheit der Gesellschafter den Verkauf auch mit der Zielsetzung, sich bei dem erwerbenden MVZ unter Verzicht auf die Vertraqsarztzulassung anstellen zu

$13 \mathrm{BGH}$, Urteil vom 22.07.2002, Az.: II ZR 90/ $01=$ BGHZ 151, 389-398, juris Rn. 13 m.w.N. 14 Ebd. lassen, ist dieser Entscheidung immanent, dass die verkaufswilligen Gesellschafter aus der BAG und damit i. d. R. auch der GbR regelmäßig ausscheiden wollen. Insofern wird man in dem Beschluss, die Praxis zu veräußern, regelmäßig auch eine gleichzeitige Kündigung der Gesellschaft von Seiten der ausscheidenden Gesellschafter erblicken müssen. Soweit im Gesellschaftsvertrag eine Nachfolgeklausel vereinbart ist, hat diese Kündigung der Gesellschaft allerdings lediglich ein Ausscheiden der verkaufswilligen Gesellschafter zur Folge, ohne dass dies die Beendigung der BAG nach sich zieht. Auf die Schutzwürdigkeit der BAG wirkt sich der Mehrheitsbeschluss über die Praxisveräußerung somit nicht aus, da die gesellschaftsvertraglich vereinbarte Nachfolgeklausel diese in jedem Falle in ihrem Bestand erhält.

Unter Beachtung der vorstehend dargestellten Grundsätze ist davon auszugehen, dass ein derartiger Mehrheitsbeschluss in unzumutbarer Weise in die Rechte der überstimmten Gesellschafter eingreift, welche den Willen haben, an der BAG festzuhalten. Selbst wenn eine Erlösbeteiligung aller Gesellschafter, d. h. auch der verkaufsunwilligen Minderheit, erfolgt, so wird dieser finanzielle Vorteil den erlittenen Nachteil des Verlustes der Betriebs- und Arbeitsmittel im Ergebnis nicht ausgleichen können. Denn dieser wird voraussichtlich nicht ausreichen, um den Aufbau einer neuen beruflichen Existenz für den einzelnen Gesellschafter zu ermöglichen. Die darin liegende Schwere des Eingriffs in die Berufsausübungsfreiheit der verkaufsunwilligen Radiologen spricht selbst vor dem Hintergrund, dass auch die überstimmten Gesellschafter sich einer ggf. vorhandenen Mehrheitsklausel im Gesellschaftsvertrag zuvor freiwillig unterworfen haben, für das Fehlen einer materiellen Legitimation und damit für eine Unwirksamkeit des Mehrheitsbeschlusses. Schließlich ist auch nicht ersichtlich, welches überwiegende Interesse der GbR bzw. BAG als solcher die derart gravierende Beschneidung der Minderheitenrechte rechtfertigt. Denn der Verkauf der Praxis liegt letztlich allein im Interesse der verkaufswilligen Gesellschafter. Ein aus dem Verkauf erwachsendes schützenswertes Interesse der Gesellschaft, z. B. deren Funktionsfähigkeit, ist indes mitnichten erkennbar. Im Gegenteil wird doch die
Funktionsfähigkeit durch den Verkauf der Praxis gerade in eklatanter Weise gestört.

Letztlich wird sogar - was allenfalls für die Bestimmung der Darlegungs- und Beweislast von Relevanz ist, wie oben dargestellt - eine Berührung des „Kernbereichs“ der Gesellschafterrechte anzunehmen sein, wenn eine Minderheit gezwungen würde, den Verkauf der gemeinsam betriebenen Radiologiepraxis ohne explizite Einwilligung hinzunehmen. Zum Kernbereich der Gesellschafterrechte zählt man jene individuellen Rechte, welche unter Berücksichtigung der Besonderheiten des jeweiligen Gesellschaftsvertrages $^{15}$ als so fundamental für die Gesellschafterposition angesehen werden, dass auf die Stimmabgabe eines jeden Gesellschafters entweder gar nicht ${ }^{16}$ oder aber jedenfalls nur mit dessen Zustimmung ${ }^{17}$ verzichtet werden kann. Von einer „antizipierten Zustimmung“ des nicht im Rahmen des Beschlusses zustimmenden Gesellschafters durch freiwillige Unterwerfung unter die im Vertrag enthaltene Mehrheitsklausel kann man nur dann ausgehen, wenn diese Klausel sich eindeutig auf diesen Eingriff bezieht und Art und Ausmaß exakt erkennen lässt. ${ }^{18}$ Auch in diesem Zusammenhang zeigt sich, dass es maßgeblich auf die konkrete Regelung zur rechtlichen Beurteilung des Mehrheitsbeschlusses im Einzelfall ankommt. Einen Eingriff in den Kernbereich der Mitgliedschaftsrechte der überstimmten Gesellschafter stellt unter anderem dar, wenn die Mehrheit eine Zweckänderung (vgl. §33 Abs. 1 S. 2 BGB) $)^{19}$ oder Auflösung ${ }^{20}$ der Gesellschaft beschließt. Die hier streitige Konstellation ist von ihrer Intensität vergleichbar. Denn in Folge der Veräußerung wäre es den verkaufsunwilligen Gesellschaftern letztlich in tatsächlicher Hinsicht unmöglich, ihren Beruf weiter auszuüben. Ebenso ist in dieser besonderen Konstellation er-

15 Schäfer in: Münchener Kommentar zum BGB, 7. Auflage 2017, § 709 Rn. 93 m.w.N.; BGH, „OTTO“ (Fn. 2), juris Rn. 15.

16 Früher, z. B.: BGH, Urteil vom 14.05.1956, Az.: II ZR 229/54 = BGHZ 20, 363-371, juris.

17 In jüngerer Rechtsprechung, z. B. BGH, Urteil vom 05.11.1984, Az.: II ZR 111/84 = NJW 1985, 974-975, juris; Schäfer, a. a. O., Rn. 91.

18 Schäfer, a. a. O., Rn. 92 m.w.N.

19 Schäfer, a.a. O., Rn. 93 m.w.N.

20 Ebd. 
neut zu bedenken, dass die Entscheidung der verkaufsunwilligen Gesellschafter zur Berufsausübung in der BAG einem besonderen grundrechtlichen Schutz unterliegt (s. o.). Die Ausübung der vertragsärztlichen Tätigkeit in einer gemeinsam geführten Praxis stellt jedoch gerade den Gesellschaftszweck dar, dessen Erreichung mit dem Verkauf der Praxis faktisch unmöglich gemacht wird.

In prozessualer Hinsicht ist zu beachten, dass zur Geltendmachung dieser Unwirksamkeit die Erhebung einer allgemeinen Feststellungsklage vor den ordentlichen Gerichten gemäß § 256 ZPO erforderlich ist. Im Zuge dieses Klageverfahrens kann das Ziel verfolgt werden, festzustellen, dass aus dem Mehrheitsbeschluss kein Rechtsverhältnis für die Minderheitengesellschafter erwächst.

\section{Aufnahme einer Klausel in den Gesellschaftsvertrag}

Es wird darauf hingewiesen, dass eine Einordnung der Problematik eines mehrheitlich gefassten Veräußerungsbeschlusses schon aufgrund der verschiedenen Möglichkeiten der gesellschaftsvertraglichen Regelungen an einiger Unübersichtlichkeit leidet und im Rahmen dieses Beitrages nicht erschöpfend dargestellt werden kann. Gerade aufgrund dieser von Fall zu Fall wohl unterschiedlich ausfallenden juristischen Bewertung empfiehlt es sich, vorsichtshalber eine Klausel in den Gesellschaftsvertrag aufzunehmen, welche einen Mehrheitsbeschluss bezüglich des Verkaufs der Gemeinschaftspraxis explizit ausschließt. ${ }^{21}$ Diese könnte den Wortlaut haben:

„Die Veräußerung der Praxis als Sachgesamtheit bedarf eines einstimmigen Beschlusses aller vorhandenen Gesellschafter; eine Beschlussfassung durch Mehrheitsbeschluss über diesen Gegenstand ist unwirksam. “

Eine solche Vertragsklausel hätte zur Folge, dass die Interessen aller Gesellschafter in jedem Falle hinreichend Berücksichtigung finden.

21 Jedenfalls wird dies naheliegend sein, sofern sich im konkreten Fall ein Verkaufswille von Gesellschaftern bei Abschluss des Gesellschaftsvertrages bereits ausmachen lässt.

\section{Vertragsarztrechtliche Aspekte}

Aus vertragsarztrechtlicher Perspektive stellt sich insbesondere die Frage des Verbleibs der Vertragsarztzulassungen der verkaufswilligen Gesellschafter. Diese werden aus der BAG ausscheiden wollen, wenn sie sich für eine Anstellung in dem die Praxis erwerbenden MVZ entscheiden.

Die Vertragsarztzulassung ist dem jeweiligen Vertragsarzt als natürliche Person im Wege einer öffentlich-rechtlichen Statusentscheidung zugeordnet. Sie wird ihm gemäß § 95 Abs. 1 S. 5 SGB V, § 24 Abs. 1 Ärzte-ZV persönlich für den Ort der Niederlassung (sog. Vertragsarztsitz) erteilt. Insofern bestimmt auch der §33 Abs. 2 ÄrzteZV folgerichtig, dass bei einer BAG die Zulassungen mehrerer Ärzte im Falle einer örtlichen BAG an einem gemeinsamen Vertragsarztsitz liegen (S. 1); dagegen liegen sie bei einer überörtlichen BAG (üBAG) an unterschiedlichen Vertragsarztsitzen (S. 2).

Insofern ist ein verkaufswilliger, aus der BAG ausscheidender Gesellschafter im Grundsatz nicht gehindert, seine ihm persönlich erteilte Vertragsarztzulassung (bzw. den persönlich erteilten Vertragsarztsitz) „mitzunehmen“ und in eine andere BAC einzutreten oder aber gemäß § 103 Abs. 4a SGB $V$ auf seine Vertragsarztzulassung zu verzichten, um sich bei dem erwerbenden MVZ - jedenfalls beabsichtigt für eine Dauer von mindestens drei Jahren ${ }^{22}$ - anstellen zu lassen. Gerade letztere Variante liegt für die hier in Frage stehende Sachverhaltskonstellation nahe.

\section{Pflicht zur Ausschreibung im Gesellschaftsvertrag}

In Ausnahmefällen kann den verbleibenden Gesellschaftern einer BAG das Recht zukommen, von den Ausscheidenden zu fordern, den Verzicht auf die Zulassung gegenüber der Kassenärztlichen Vereinigung zu erklären und einen Antrag auf Ausschreibung des frei werdenden Vertragsarztsitzes im Nachbesetzungsverfahren zu stellen (nach §104 Abs. 4 S. 1 SGB V). Hierfür

22 Vgl. BSG, Urteil vom 04.05.2016, Az.: B 6 KA $21 / 15 R=$ BSGE 121, 143-154, juris Rn. $28 \mathrm{f}$. bedarf es jedoch stets zunächst einer entsprechenden Klausel im Gesellschaftsvertrag, welche dieses Recht gewährt.

Wurde eine derartige Klausel zwischen den BAG-Mitgliedern vereinbart, darf diese nicht wegen Sittenwidrigkeit (§138 BGB) nichtig sein. In diesem Falle kollidiert die durch Art. 12 Abs. 1 GG geschützte Berufsfreiheit des Ausscheidenden mit jener der verbleibenden Ärzte der BAG, welche ein Interesse daran haben, die BAG im bestehenden - und genehmigten - Umfang fortzuführen. Dieser Konflikt der widerstreitenden Interessen ist nach der Rechtsprechung mittels einer interessengerechten Abwägung (sog. Grundsatz der praktischen Konkordanz) zu lösen, woraus sich für die in Frage stehende Verzichtsklausel einschränkende Wirksamkeitsvoraussetzungen ergeben.

Nach der Rechtsprechung des Bundesgerichtshofs ist bei einer derartigen Konfliktlage dem Erhalt der Gemeinschaftspraxis bzw. BAG temporär der Vorrang einzuräumen. Deswegen könne ein Verzicht auf die Vertragsarztzulassung und Ausschreibung im Nachbesetzungsverfahren von einem ausscheidenden Gesellschafter zumindest dann gefordert werden, wenn der Ausscheidende zum einen eine vakant gewordene Vertragsarztstelle in der BAG übernommen hatte und zum anderen, wenn er dies erst vor so kurzer Zeit getan hat, dass er die BAG durch seine Tätigkeit noch nicht entscheidend mitprägen konnte. ${ }^{23}$ Der Bundesgerichtshof führt dazu aus:

"Jede Aufnahme eines Partners in die Praxis würde zum unkalkulierbaren Risiko, könnte der ausscheidende Arzt seine Zulassung mit der Folge des Verlustes des Vertragsarztsitzes für die aufnehmende Praxis nach derartig kurzer Zeit einfach mitnehmen. Anders mögen die Dinge allenfalls dann liegen, wenn aus Gründen, für die der aufnehmende Arzt verantwortlich ist, der weitere Verbleib in der Praxis für den Aufgenommenen unzumutbar ist. "24

23 BGH, Urteil vom 22.07.2002, Az.: II ZR 90/01 (Fn. 13), juris Rn. 17f; Urteil vom 22.07.2002, Az.: II ZR 265/00 = NJW 2002, 3538-3539, juris Rn. 16.

24 BGH, Urteil vom 22.07.2002, Az.: II ZR 265/00 (Fn. 23), juris Rn. 16. 
Zu der sich hieran anschließenden Fragestellung, ab welchem konkreten Zeitraum der Tätigkeit in der BAG denn eine entscheidende Mitprägung anzunehmen ist, hat der Bundesgerichtshof leider bisher keine exakte zeitliche Grenze benannt. Er hat in dem entschiedenen Fall allerdings angenommen, dass ein Arzt, welcher für einen Zeitraum von unter zwei Jahren der BAG zugehörig war, diese noch nicht entscheidend mitgeprägt habe. Das vertraglich vereinbarte Recht der verbleibenden Gesellschafter, vom Ausscheidenden den Verzicht auf die Zulassung und die Antragstellung auf Ausschreibung zu fordern, wurde für ihn somit als zumutbare Einschränkung seiner Berufsausübungsfreiheit erachtet und war deswegen auch nicht wegen Sittenwidrigkeit nichtig. ${ }^{25}$

Insofern wird man, selbst wenn der Bundesgerichtshof sich zu einer konkreten zeitlichen Grenze bisher nicht abschließend geäußert hat, jedenfalls davon ausgehen können, dass eine entscheidende Mitprägung eines neu eintretenden Gesellschafters in eine BAG ab einem Zeitraum der Tätigkeit von drei Jahren gegeben sein wird.

\section{Fazit}

Beschließt die Mehrheit der Gesellschafter einer BAG, dass die geführte Gemeinschaftspraxis insgesamt veräußert werden soll, sind den überstimmten Gesellschaftern zumindest in begrenztem Umfang
Möglichkeiten an die Hand gegeben, die BAG weiterzuführen oder aber die Veräußerung zu verhindern. Diese Möglichkeiten sind in jedem Falle jedoch primär abhängig von der konkreten Ausgestaltung der gesellschaftsvertraglichen Regelungen.

Einerseits ist es unter Umständen empfehlenswert, im Zuge der Prävention und zur Verhinderung von Unsicherheiten im Hinblick auf die Auslegung des Gesellschaftsvertrages eine explizite Ausschlussregel in den Vertrag aufzunehmen, welche es verbietet, dass lediglich die Mehrheit der insgesamt vorhandenen Gesellschafter in der Lage ist, eine Veräußerung der Gemeinschaftspraxis vorzunehmen.

Ist eine derartige Klausel im Vertrag - was wohl die Regel sein wird - nicht vorhanden, sondern im Gegensatz dazu eine Vertragsbestimmung, welche explizit oder nach Auslegung (ggf. unter Zuhilfenahme außerhalb des Vertrages liegender Umstände) einen Mehrheitsbeschluss bezüglich der Praxisveräußerung ausdrücklich als zulässig bestimmt, ist die formelle Legitimation einer mehrheitlichen Verkaufsentscheidung zwar gegeben. Es sprechen dann allerdings gute Gründe dafür, eine Unwirksamkeit des Mehrheitsbeschlusses wegen fehlender materieller Legitimation anzunehmen. Denn die verbleibenden Gesellschafter werden in unzumutbarer Weise in ihrer Berufsausübungsfreiheit aus Art. 12 Abs. 1 GG eingeschränkt, ohne dass ihnen im Gegen- zug ein äquivalenter Vorteil zufließt. Die Unwirksamkeit des Beschlusses kann im Wege der allgemeinen Feststellungsklage gemäß § 256 ZPO zivilgerichtlich geltend gemacht werden.

Andererseits kann eine BAG ihren Bestand dadurch schützen, indem eine Verzichtsklausel bezogen auf die Vertragsarztzulassung der ausscheidenden, verkaufswilligen Gesellschafter in den Gesellschaftsvertrag aufgenommen wird. Diese ist jedoch nicht zeitlich unbegrenzt wirksam, sondern nur solange, wie die ausscheidenden Gesellschafter erst für eine so kurze Zeit in der BAG tätig waren, dass sie diese nicht entscheidend mitgeprägt haben. Dies wird man in der Regel für einen Zeitraum von maximal drei Jahren ab Eintritt in die Gesellschaft annehmen können.

Prof. Dr. Peter Wigge

Rechtsanwalt

Fachanwalt für Medizinrecht

Philip Steuwer

Rechtsanwalt

Rechtsanwälte Wigge

Scharnhorststraße 40

48151 Münster

Telefon: (0251) 53 595-0

Telefax: (0251) 53 595-99

E-Mail: kanzlei@ra-wigge.de

www.ra-wigge.de 